\title{
Bunch Length Measurements at the Advanced Photon Source (APS) Linear Accelerator *
}

\author{
N. S. Sereno, R. Fuja, C. Gold, A. E. Grelick, A. Nassiri, J. J. Song, M. White \\ Advanced Photon Source, Argonne National Laboratory \\ 9700 South Cass Avenue, Argonne, Illinois 60439 USA
}

\begin{abstract}
Measurements of the APS linac micro-bunch length are performed by backphasing a single 2856-MHz, S-band linac waveguide and using a downstream spectrometer to observe the beam. By measuring the beam width in the dispersive plane as a function of $\mathrm{rf}$ power into the linac waveguide, the bunch length can be determined absolutely provided the beam energy and dispersion at the spectrometer are known. The bunch length determined in this fashion is used to calibrate a fifth-harmonic bunch length cavity which is used for real-time bunch length monitoring.
\end{abstract}

\section{INTRODUCTION}

Bunch length measurements of the APS linac electron beam are required in order to evaluate the performance of the low energy bunching process of the beam emitted by the thermionic gun. Bunching of the $100-\mathrm{keV}$ beam is performed by a standing wave cavity and drift (the prebuncher) followed by a 5-cell traveling wave cavity (the buncher) where each cavity operates at a frequency of $2856 \mathrm{MHz}$. After the buncher, the beam is accelerated from an energy of $1.4 \mathrm{MeV}$ to $50 \mathrm{MeV}$ by the first $3-\mathrm{m}, 86-$ cell accelerating waveguide operating at $2856 \mathrm{MHz}$. Four downstream accelerating waveguides further accelerate the beam to $220 \mathrm{MeV}$ at the positron target. After the target, nine accelerating waveguides are used to accelerate the positrons to $450 \mathrm{MeV}$.

The electron beam consists of 30-ns macropulses repeated at a $2-\mathrm{Hz}$ rate. Each 30 -ns macropulse consists of 86 micro-bunches after the bunching process is completed (essentially after the beam passes the buncher). The microbunch length of the APS linac electron beam (from now on referred to as the bunch length) has been measured by backphasing a single accelerating waveguide and observing the beam after a downstream spectrometer. The accelerating waveguide is phased so that the centroid of each microbunch passes through it when the electric field is zero (zero crossing). Energy spread is induced in the micro-bunch because at zero crossing, depending on the slope of the rf waveform, particles obtain more or less energy depending on their location within the micro-bunch. The induced energy spread is linearly related to the bunch length for bunches that are short compared to the rf period (350 ps at $2856 \mathrm{MHz}$ ).

\footnotetext{
*Work supported by U.S. Department of Energy, Office of Basic Energy Sciences, under Contract No. W-31-109-ENG-38
}

A standing wave cavity operating at the fifth harmonic of the bunching frequency $(14.28 \mathrm{GHz})$ is used for real time monitoring of the bunch length in the linac [1]. The peak output power of the cavity depends on the bunch length. The fifth harmonic frequency was chosen to maximize the output power for small ( $\sim 5$ ps rms) micro-bunches consistent with mechanical and electrical constraints. Calibration of the cavity is accomplished by first measuring the bunch length by the backphasing technique and using the result to determine the effective cavity shunt impedance.

\section{THEORY}

The basic apparatus for the bunch length measurement consists of an accelerating waveguide through which a relativistic bunched electron beam passes on zero crossing. The beam is subsequently observed-downstream of the accelerating waveguide at a spectrometer consisting of a dipole magnet, a drift, and a screen. The transverse position of a given beam particle in the dispersive plane on the screen is given by

$$
x=x_{\beta}+\eta \frac{\delta p+\left(E_{g} / c\right) \sin \phi}{p}
$$

where $x_{\beta}$ is the usual betatron oscillation of the particle, $\eta$ is the dispersion at the viewscreen in meters, $\delta p$ is the intrinsic momentum offset of the particle, $p$ is the central momentum of the beam, $E_{g}$ is the maximum energy gain in $\mathrm{MeV}$ of a particle that traverses the accelerating waveguide on crest, and $\phi$ is the phase of the particle in radians relative to the particle that goes through the accelerating waveguide on zero crossing. Equation (1) shows that the transverse position of the particle is linearly related to the phase offset from zero crossing for $\phi<<1$ radian.

For a given particle distribution for each micro-bunch, Eq. (1) can be used to write the rms beam size in terms of parameters of the backphased waveguide and spectrometer. The rms size is defined by

$$
\sigma^{2} \equiv\left\langle(x-\langle x\rangle)^{2}\right\rangle
$$

where \langle\rangle denotes integration of the particle coordinates over the particle distribution. Inserting Eq. (1) into Eq. (2) results in

$$
\begin{aligned}
\sigma^{2}= & \sigma_{\left(x_{\beta}+D \frac{\delta_{p}}{p}\right)}^{2}+\left\{\left\langle x_{\beta} \sin \phi\right\rangle-\left\langle x_{\beta}\right\rangle\langle\sin \phi\rangle+\right. \\
& \left.\frac{\eta}{p}(\langle\delta p \sin \phi\rangle-\langle\delta p\rangle\langle\sin \phi))\right\} \frac{2 \eta}{p c} E_{g}+ \\
& \frac{\eta^{2} \sigma_{\sin \phi}^{2}}{(p c)^{2}} E_{g}^{2}
\end{aligned}
$$




\section{DISCLAIMER}

This report was prepared as an account of work sponsored by an agency of the United States Government. Neither the United States Government nor any agency thereof, nor any of their employees, makes any warranty, express or implied, or assumes any legal liability or responsibility for the accuracy, completeness, or usefulness of any information, apparatus, product, or process disclosed, or represents that its use would not infringe privately owned rights. Reference herein to any specific commercial product, process, or service by trade name, trademark, manufacturer, or otherwise does not necessarily constitute or imply its endorsement, recommendation, or favoring by the United States Government or any agency thereof. The views and opinions of authors expressed herein do not necessarily state or reflect those of the United States Government or any agency thereof. 


\section{DISCLAIMER}

Portions of this document may be illegible in electronic image products. Images are produced from the best available original document. 


$$
\sigma_{\sin \phi}^{2} \equiv\left\langle(\sin \phi-\langle\sin \phi\rangle)^{2}\right\rangle .
$$

In Eq. (3), the first term is due to the natural emittance and energy spread of the beam. The second term results from correlations between the particle betatron oscillation, phase relative to zero crossing and natural momentum spread. The third term contains the rms bunch length when

$$
\sigma_{\sin \phi} \simeq \sigma_{\phi},
$$

which is an excellent approximation for short bunches where $\sin \phi \simeq \phi$. Typical rms bunch lengths for the APS linac are $\sim 5^{\circ}$. and therefore meet the approximation given by Eq. 5 (for comparison $1^{\circ}=1.03$ ps for $2856 \mathrm{MHz}$ ).

\section{MEASUREMENT RESULTS}

Equation (3) indicates that the rms bunch length can be obtained by fitting a quadratic curve to a measurement of $\sigma^{2}$ vs $E_{g}$. The form the fitting function therefore takes is

$$
\sigma^{2}=a_{\diamond}+a_{1} E_{g}+a_{2} E_{g}^{2},
$$

where the above terms are readily identified with those of Eq. (3). The second-order term yields the micro-bunch length provided the central momentum (energy) and dispersion at the viewscreen are known. It is desireable to go to a large waveguide energy gain so that the quadratic term dominates the other two. The relative error in the quadratic term will therefore be minimized.

Figure 1 shows the data taken using a single accelerating waveguide driven by a single klystron. The beam energy at the entrance to the accelerating waveguide was 220 $\mathrm{MeV}$. The data were taken at a beam current of $150 \mathrm{~mA}$ average current per macropulse ( $52 \mathrm{pC}$ per micro-bunch) due to the fact that camera and viewscreen saturation effects limit resolution at higher beam currents. The microbunch length backphase measurement was performed using the beam resulting from a "standard" setup for the buncher and prebuncher. The resulting beam spot distribution on the spectrometer viewscreen was symmetric and easily analyzed. The beam image analysis software computes $\sigma$ directly from the measured beam intensity distribution according to Eq. (2). No assumptions about the exact microbunch structure need be made. At each accelerating waveguide energy gain, the rms size was taken to be the average of the rms size of five beam snapshots taken. The error bars are taken to be the standard error for the mean [2]. This averaged out shot-to-shot fluctuations of the rms size due to rf and other noise sources. The quadratic fit to the data is shown as the solid line. The fit is acceptable with most data points lying within a distance of twice their error bar of the fit.

Table 1 summarizes the measurement parameters, the results of the fit, and gives the inferred bunch length. The dominant errors contributing to the bunch length error are the error in the second term $a_{2}$ and the dispersion. The last entry in Table 1 is an estimate of the FWTM (full width at $10 \%$ of the maximum height of the distribution) bunch length. The FWTM bunch length was estimated by

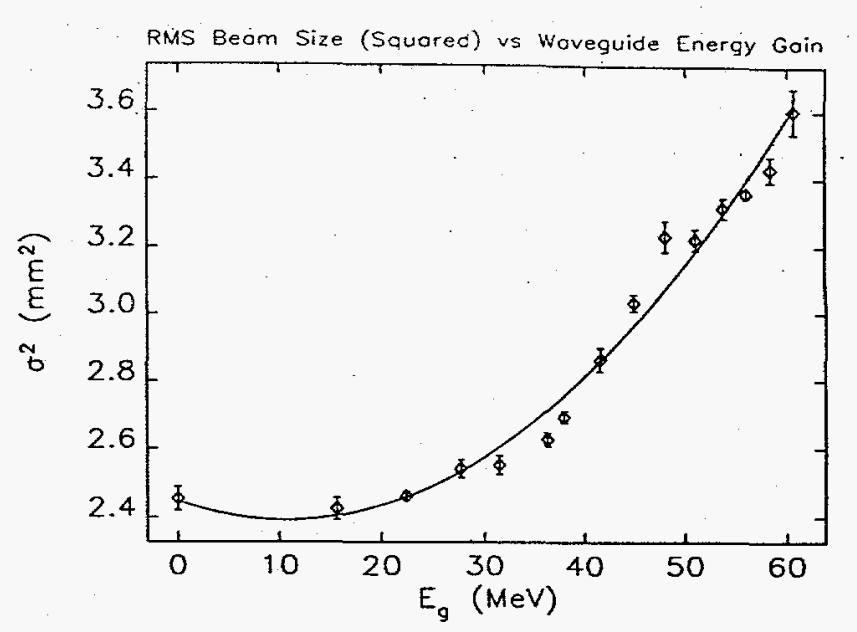

Figure 1: Bunch length measurement data consisting of $\sigma^{2}$ vs $E_{g}$. The solid line shows the best fit quadratic curve to the data.

Table 1: Bunch length measurement parameters.

\begin{tabular}{|c|c|}
\hline Parameter & Value \\
\hline$p$ & $334.8 \pm 3.3 \mathrm{MeV}$ \\
$\eta$ & $194 \pm 5 \mathrm{~mm}$ \\
$a_{0}$ & $2.45 \pm .05 \mathrm{~mm}^{2}$ \\
$a_{1}$ & $(-1.0 \pm .3) \times 10^{-2} \mathrm{~mm}^{2} / \mathrm{MeV}$ \\
$a_{2}$ & $(4.9 \pm .5) \times 10^{-5}(\mathrm{~mm} / \mathrm{MeV})^{2}$ \\
$\sigma_{\phi}$ & $(2.2 \pm .1)^{\circ}$ \\
FWTM $_{\phi}$ & $(9.9 \pm .6)^{\circ}$ \\
\hline
\end{tabular}

comparing the ratio of the FWTM beam size to $\sigma$ for each data point and taking the average of this ratio for all the data points. The average ratio was found to be 4.53 (for comparison, the ratio of the FWTM size and the rms size $\sigma$ for a Gaussian distribution is 4.3).

\section{FIFTH HARMONIC CAVITY CALIBRATION}

The measurement procedure just described, though automated using the SDDS tools [3], is time consuming. A bunch monitor previously described [1] is used for real-time (shot-to-shot) bunch length monitoring. The peak cavity output power for a given beam current is given by

$$
P=\frac{R I_{\circ}^{2}}{2} e^{-m^{2} \sigma_{\phi}^{2}}
$$

where $I_{0}$ is the average beam current and the notation for the effective shunt impedance $R$ from reference [1] is kept. The power given by Eq. (7) is seen to depend on the bunch form factor $e^{-m^{2} \sigma_{\phi}^{2}}$. This form factor arises specifically because a Gaussian shape for the microbunch was assumed. For short bunches, the precise functional form of the form factor matters little because the second order Taylor expansion to second order for different form factors is identical for frequencies that are small relative to the rolloff frequency given by the inverse temporal bunch length. The 
factor multiplying the rms bunch length $m$, is the harmonic number of the bunched beam signal. Since the fundamental cavity mode operates at the fifth harmonic of the bunching frequency $m=5$ for the cavity considered here.

The reason for going to as large a harmonic number as possible is to increase measurement sensitivity to short bunches. Equation (7) is now used to estimate the smallest bunch length measurable for a cavity (or any detector) operating at some harmonic of the bunching frequency. Assuming that $0.1-\mathrm{dB}$ power changes are the minimum detectable in the presence of typical noise sources, the minimum rms bunch length is given by

$$
\sigma_{\phi}^{\min }=\frac{1}{10 \sqrt{\log (e)}} \frac{1}{m}
$$

which for our cavity turns out to be $1.74^{\circ}\left(7.48^{\circ} \mathrm{FWTM}\right.$ assuming a Gaussian distribution). Inspection of Table 1 reveals that the bunch length determined in the backphase measurement is nearly at the theoretical lower limit defined by Eq. (8).

Calibration of the cavity consists of determining the effective shunt impedance in Eq. (7). The peak cavity output power was measured using a calibrated fast diode. Corrected for cable losses, the peak cavity output power was measured to be $45.9 \mathrm{~mW}$ for a beam current of $150 \mathrm{~mA}$. Using the measured bunch length listed in Table 1 and Eq. (7), the shunt impedance turns out to be $4.2 \Omega$. The calculated shunt impedance from SUPERFISH is $33 \Omega$. The order of magnitude difference stems from two primary effects. The first is that for a $30-n s$ pulse, the cavity is not completely filled, and second, the loaded and unloaded $Q$ results in a mismatch which reduces the peak output power [4]. Both these effects increase the shunt impedance by a factor of two to three.

Further studies are being conducted by varying the beam current to get a more precise value for the shunt impedance and to evaluate the measurement uncertainties involved. A measurement uncertainty of $10 \%$ for the shunt impedance should be adequate and achievable. One way of determining the shunt impedance would be to produce a bunch length smaller than the minimum detectable as given by Eq. (8) and measure the peak power as a function of beam current squared. The slope of this curve from Eq. (7) is simply $R / 2$. Bunch lengths greater than that given by Eq. (8) would manifest themselves as a reduction in the slope given by the form factor in Eq. (7). Of course, this measurement of $R$ assumes that the bunch length does not vary appreciably as the beam current is varied. This is a good assumption at the very low beam currents used here, where space charge is negligible. Extention of this idea would require a separate bunch length measurement using the backphase technique at each beam current.

\section{CONCLUSION}

The bunch length has been measured by backphasing a single accelerating waveguide and observing the beam at a downstream spectrometer. Improvements to the measurement include going to higher values of $E_{g}$ by using four backphased waveguides driven by a single klystron and SLED [5]. Compared to a single accelerating waveguide, four accelerating waveguides would allow four times the energy spread to to be induced in the beam for a given bunch length. Another approach would be to increase the dispersion of the spectrometer which would require hardware modification.

The fifth-harmonic cavity calibration was done at a single beam current and bunch length. Additional measurements will be made at multiple beam currents and bunch lengths to check the calibration over a broad range of beam parameters. The measured cavity shunt impedance agrees with the calculation when the effects of cavity filling during the beam macropulse and mismatch are taken into account.

\section{ACKNOWLEDGEMENTS}

The authors would like to thank all those at the APS involved in linac commissioning for their efforts. The first author would like to thank G. Krafft and B. Bowling at CEBAF for their comments and suggestions regarding this measurement.

\section{REFERENCES}

[1] A. Nassiri and A. Grelick, "A Fifth Harmonic RF Bunch Monitor for the ANL-APS Electron Linac," Proceedings of the 1993 Particle Accelerator Conference, Washington DC, 2412 (1993).

[2] P. R. Bevington, Data Reduction and Error Analysis for the Physical Sciences, McGraw-Hill Book Co. 70 (1969).

[3] M. Borland, "A Self-Describing File Protocol for Simulation Integration, and Shared Post-Processing," these proceedings.

[4] D. A. Goldberg and G. R. Lambertson, AIP Conference Proceedings, 249, 560 (1992).

[5] Z. D. Farkas, H. A. Hogg, G. A. Loew, and P. B. Wilson, "SLED: A Method of Doubling SLAC's Energy," SLAC-PUB-1453, June 1974. 